\title{
Efficient Two-Move Blind Signatures in the Common Reference String Model
}

\author{
E. Ghadafi and N.P. Smart \\ Dept. Computer Science, \\ University of Bristol, \\ Merchant Venturers Building, \\ Woodland Road, \\ Bristol, BS8 1UB, \\ United Kingdom. \\ \{ghadafi,nigel\}@cs.bris.ac.uk
}

\begin{abstract}
Blind signatures provide a mechanism for achieving privacy and anonymity whereby a user gets the signer to sign a message of his choice without the signer learning the content of the message, nor linking message/signature request pairs when he sees the final signature. In this paper, we construct a blind signature that requires minimal interaction (two moves) between the user and the signer, and which results in a signature which is a signature with respect to a standard (i.e. non-blind) signature scheme. The signature request protocol is akin to the classic, blind-unblind methodology used for RSA blind signatures in the random oracle model; whilst the output signature is a standard CamenischLysyanskaya signature in bilinear groups. The scheme is secure in the common reference string model, assuming a discrete logarithm related assumption in bilinear groups; namely a new variant of the LRSW assumption. We provide evidence for the hardness of our new variant of the LRSW by showing it is intractable in the generic group model.
\end{abstract}

\section{Introduction}

BACKGROUnD: Since their introduction by Chaum [11], blind signatures have been used in a number of cryptographic applications that require one party (a signer) to authenticate a message for another party (the user), whilst maintaining privacy of the user's message. The classic example of their use is in e-cash protocols [11] where a bank acts as the signer, and the message is a representation of digital cash; the privacy requirement comes from the non-traceability requirement of cash.

A blind signature must satisfy two security requirements [20, 27], blindness and unforgeability. By blindness we mean that the signer does not learn what message he has signed and in addition, when he later sees the message at the verification process, he cannot link it to its corresponding 
signature request. Unforgeability on the other hand guarantees that the user cannot output any new signatures that he has not asked the signer to sign for him, or in other words, the number of signatures the user can compute is equal to the number of completed interactions he was involved in with the signer.

Since their introduction, a number of authors have presented blind signature algorithms based on different assumptions and in different models. For example, schemes based on factoring related assumptions have been given in the Random Oracle Model (ROM) [3], and in the Common Reference String (CRS) model [9]; schemes based on discrete logarithm related assumptions have been given in the ROM $[1,5,27]$ and in the CRS model $[2,14,25]$; schemes based on a combination of discrete logarithm and factoring based assumptions have been given in the CRS model [21]; finally, in $[13,20]$ schemes in the CRS model are given under general assumptions.

A blind signature consists of two distinct phases. In the first phase, which we shall call the signature request phase, the user obtains from the signer the signature on the message he requires. In the second phase, the signature and message are made public and anyone can apply the public verification algorithm to verify the message/signature pair. The signature request phase is the most complex of all phases. One could consider such a phase as a general secure two-party computation, where the user has the message as his private input, whereas the signer has a secret key as his private input. After such a secure computation, the user outputs a valid blind signature on his secret message.

The "classic" blind signature schemes are in the ROM and are essentially Full-Domain-Hash (FDH) style signature schemes. In these schemes, the hash function is applied to the message, the result is then blinded and sent to the signer. The signer signs the blinded message as he would sign a normal message. On receiving the output from the signer the user then unblinds the signature using a homomorphic property of the underlying signature scheme. Such a mechanism is the basis of the original RSA based scheme of Chaum [11], which was proved secure in [3]; Chaum's scheme outputs standard RSA-FDH signatures [4]. It also forms a basis of the BLS signature [7] based blind signature scheme of Boldyreva [5]. The advantage of such schemes is that the output signature corresponds to a standard signature scheme; in these two cases RSA-FDH and BLS respectively. Our construction has a similar flavour in that the signature generation protocol is of the blind/unblind variant and that the output signature is a "standard" signature, namely a Camensich-Lysyanskaya 
(CL) signature [10]. However, we dispense with the need for random oracles and instead work in the CRS model.

PRIOR WORK IN THE CRS MODEL: Due to the round-optimal nature of a two-move signature request phase, and the desire to avoid the use of the random oracle, much recent work has focused on developing roundoptimal blind signatures in the CRS model.

In [13], Fischlin presented a scheme in the CRS model which has a two-move signature request protocol. The scheme is a generic construction from basic primitives, namely schemes for commitment, encryption and signatures as well as generic non-interactive zero knowledge (NIZK) proofs for NP-languages. The signature request protocol consists of the user sending a commitment to the message to the signer, who responds with a signature on the commitment. The user then uses this signature on the commitment to construct the blind signature, by first encrypting the commitment and the signature, and then adding a NIZK proof that the encrypted signature is a valid signature on the encrypted commitment, and that the encrypted commitment is a commitment to the specific message.

Using the notion of automorphic signatures, Fuchsbauer [14] (see also [2]) presented a variant of the construction of Fischlin, using specific efficient components. In particular, he made use of the efficient NIZK proofs of Groth and Sahai $[18,17]$ which hold for only specific NP-statements in bilinear groups. In Fuchsbauer's scheme, the blind signature is constructed by providing a Groth-Sahai proof of knowledge of a signature on a message (as opposed to a signature on a commitment as in Fischlin's generic construction). This makes the underlying NIZK proofs simpler, but makes use of a different signature request phase. The resulting blind signature consists of around 30 group elements, and is the most efficient round-optimal blind signature scheme in the CRS model known to date.

Fuchsbauer's scheme is based on a new intractibility assumption called the ADH-SDH assumption, which he shows holds in the Generic Group Model (GGM) [29,23]. This is a falsifiable assumption, in the sense of Naor [24], which is closely related to the $q-\mathrm{SDH}$ problem lying behind the Boneh-Boyen signature scheme [6]. However, the resulting blind signature is not a standard signature, e.g. it is not a true Boneh-Boyen signature.

In this paper, we present a round-optimal blind signature scheme in the CRS model which is significantly more efficient than Fuchsbauer's scheme; a signature only consists of three group elements. Indeed the resulting signature is a standard CL signature on the message $m$. We note that our required hardness assumption, being interactive, is not falsifi- 
able. However, this property is inherited from the underlying CL signature where the underlying hardness assumption is the LRSW assumption, which is itself interactive.

In [16], the authors present a generic round-optimal blind signature scheme in the standard model, which results in blind signatures from any standard signature scheme. However, their construction is not as efficient as our construction in the CRS model for the CL signature scheme. In particular, the generic construction of [16] requires the use of ZAPs and two-party secure function evaluation protocols.

Our Scheme: The scheme we present has a number of similarities to previous work, yet a number of distinct advantages. As already remarked it results in standard CL signatures, is optimal in the number of moves in the signing protocol, and dispenses with the need for using the ROM.

Recall that security of the RSA-FDH based blind signature is not based on the same assumption as the original RSA-FDH signature, indeed it is based on a stronger one, the same holds true for the BLS-based blind signature. In our construction the same property re assumptions holds; whilst security of the standard CL signature scheme is based on the LRSW assumption [22], the security of our scheme relies on a stronger assumption, which we call the Extended-LRSW (E-LRSW) assumption, which is itself related to the Blind-LRSW (B-LRSW) assumption previously proposed in [12]. We justify the hardness of this new assumption by presenting a proof in the GGM. We note, our proof can be modified to also justify the B-LRSW assumption in the GGM.

We note that the CRS-based scheme in [21] also outputs standard CL signatures, however, the signature request protocol requires factoringbased assumptions to provide security. Our signature request protocol is significantly simpler.

We now state some disadvantages of our scheme. Firstly, to obtain a highly efficient protocol, we work in the honestly-generated keys model; security can easily be obtained in the adversarially-generated keys model with either the addition of an extra round of communication, the addition of NIZK proofs of knowledge, or a more complex setup phase. Secondly, our scheme reduces to an interactive assumption rather than a standard cryptographic assumption. However, this is relatively standard in the construction of efficient blind signature schemes, e.g. [11,5]. It remains an open problem to derive a (truly) efficient round-optimal blind signature scheme in the CRS model which allows adversarially-generated keys, and which reduces to a non-interactive assumption. 
Paper Organization: The rest of the paper is organized as follows; In Section 2 we recap on the syntax and security definitions for blind signatures. In Section 3 we recap on the basic properties of bilinear groups that we shall need, and we will present the underlying hard problems on which the security of our scheme rests. We present our scheme in Section 4, with the security proof provided in Section 5 . Finally, we provide evidence for the hardness of our new intractability assumption in the GGM in Appendix A.

\section{Syntax and Security of Blind Signatures}

In this section we define the syntax of blind signatures that we shall use, as well as recap on the standard security model. Since we are focusing on signature request phases which are two-move, we specialise the syntax for this case. This is purely to make the description of the security model and our scheme more transparent.

Syntax: A blind signature scheme BS (with a two-move signature request phase) in the CRS model consists of six probabilistic polynomial time algorithms

$$
\mathrm{BS}=\left(\text { Setup }_{\mathrm{BS}}, \text { KeyGen }_{\mathrm{BS}}, \text { Request }_{\mathrm{BS}}, \text { Issue }_{\mathrm{BS}}, \text { Unblind }_{\mathrm{BS}}, \text { Verify }_{\mathrm{BS}}\right) .
$$

The syntax of these algorithms is defined as follows; where to aid notation all algorithms (bar Setup $\mathrm{BS}_{\mathrm{BS}}$ ) are assumed to take as implicit input $\mathrm{CRS}_{\mathrm{BS}}$ as output by Setup ${ }_{\mathrm{BS}}$;

- $\operatorname{Setup}_{\mathrm{BS}}\left(1^{\lambda}\right)$ : Takes as input a security parameter $\lambda$ and outputs a common string $\mathrm{CRS}_{\mathrm{BS}}$. We assume $\mathrm{CRS}_{\mathrm{BS}}$ contains a description of the key and message spaces for the scheme.

- KeyGen $\operatorname{BS}\left(1^{\lambda}\right)$ : Takes as input the security parameter and outputs a pair of public/secret keys $\left(\mathrm{pk}_{\mathrm{BS}}, \mathrm{sk}_{\mathrm{BS}}\right.$ ) for the signer.

- Request $_{\mathrm{BS}}\left(m, \mathrm{pk}_{\mathrm{BS}}\right)$ : This algorithm, run by the user, takes a message $m$ in the space of messages $\mathcal{M}$ and the public key $\mathrm{pk}_{\mathrm{BS}}$, and produces a signature request $\rho$, plus some state $\mathrm{St}$ (which is assumed to contain $m)$.

- Issue $\mathrm{BS}_{\mathrm{BS}}\left(\rho, \mathrm{sk}_{\mathrm{BS}}\right)$ : This algorithm, run by the signer, takes the signature request $\rho$ and the secret key $\mathrm{sk}_{\mathrm{BS}}$, and produces a pre-signature $\beta$.

- Unblind $\mathrm{BS}\left(\beta, \mathrm{St}, \mathrm{pk}_{\mathrm{BS}}\right)$ : On input of $\beta$, St and the public key $\mathrm{pk}_{\mathrm{BS}}$, this algorithm produces a blind signature $\sigma$ on $m$, or it outputs $\perp$.

- $\operatorname{Verify}_{\mathrm{BS}}\left(m, \sigma, \mathrm{pk}_{\mathrm{BS}}\right)$ : This is the public signature verification algorithm. This should output 1 if $\sigma$ is a valid signature on $m$ and 0 otherwise. 
Correctness of the blind signature algorithm is that if both parties behave honestly then signatures should verify, i.e. for all CRS's output by Setup we have,

$$
\begin{aligned}
& \operatorname{Pr}\left[\left(\mathrm{pk}_{\mathrm{BS}}, \mathrm{sk}_{\mathrm{BS}}\right) \leftarrow \operatorname{KeyGen}_{\mathrm{BS}}\left(1^{\lambda}\right), m \leftarrow \mathcal{M},(\rho, \mathrm{St}) \leftarrow \operatorname{Request}_{\mathrm{BS}}\left(m, \mathrm{pk}_{\mathrm{BS}}\right),\right. \\
& \beta \leftarrow \operatorname{Issue}_{\mathrm{BS}}\left(\rho, \mathrm{sk}_{\mathrm{BS}}\right), \sigma \leftarrow \operatorname{Unblind}_{\mathrm{BS}}\left(\beta, \mathrm{St}, \mathrm{pk} \mathrm{BS}_{\mathrm{BS}}\right): \\
& \left.\operatorname{Verify}_{\mathrm{BS}}\left(m, \sigma, \mathrm{pk}_{\mathrm{BS}}\right)=1\right]=1 .
\end{aligned}
$$

SECURITY: The standard security model for blind signatures $[20,27]$ consists of two properties, blindness and unforgeability. Intuitively, blindness says that an adversarial signer who chooses two messages $m_{0}$ and $m_{1}$ and then interacts with an honest user who requests signatures on those messages (in an order unknown to the signer), is unable to tell the order in which the messages were signed upon being presented with the final unblinded signatures. On the other hand, unforgeability deals with an adversarial user whose goal is to obtain $k+1$ distinct message/signature pairs given only $k$ interactions with the honest signer.

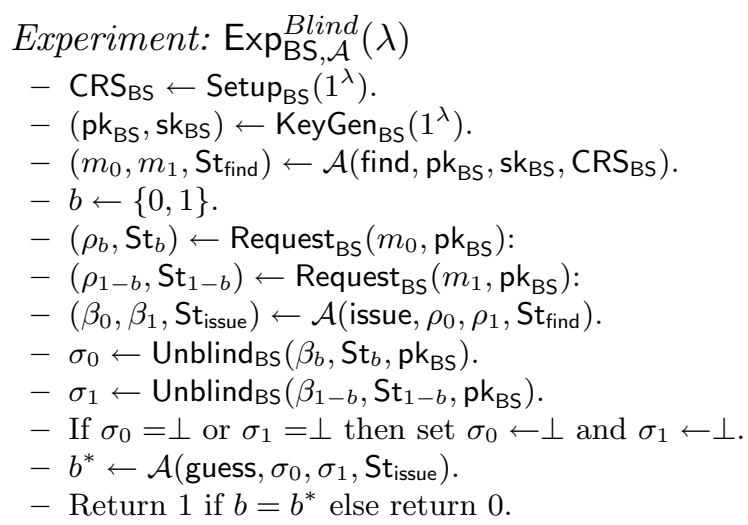

Fig. 1. The blindness experiment

To define blindness, we consider an adversary $\mathcal{A}$ which has three modes find, issue and guess, running in an experiment as in Figure 1. Note that the experiment is defined for honestly-chosen keys. Our security results will hold, re blindness, for adversarially-chosen keys as long as the challenger in the blindness game is given access to the secret key as well.

We can obtain full security against adversarially-chosen keys by simply requesting a secret key holder to prove, in zero-knowledge, knowledge of 
the underlying secret key for a given public key, and then using, within our proof for blindness, a knowledge extractor for the zero-knowledge proof to extract the witness (i.e. the secret key). The additional security obtained from adversarially-chosen keys comes however at the expense of the zero-knowledge proof. To obtain the same number of rounds, we will require such a proof to be non-interactive, and hence costly with currently known techniques; or it can be efficient and interactive, and hence cost more rounds. Another alternative would be in the setup phase for the signer to prove knowledge of their secret keys via an (interative or non-interactive) zero-knowledge proof. In the interactive case, we would however require the ability to rewind the signer to the start of the set up phase in order to extract the secret within our proof.

Our focus is on a protocol which is efficient in the honestly-generated keys model, but extending our results (admittedly with a loss of efficiency) to the adversarially-generated keys model in one of the ways described above is trivial. We define $\operatorname{Adv}_{\mathrm{BS}, \mathcal{A}}^{\text {Blind }}(\lambda)=\left|2 \cdot \operatorname{Pr}\left[\operatorname{Exp}_{\mathrm{BS}, \mathcal{A}}^{\text {Blind }}(\lambda)=1\right]-1\right|$ and we say that the scheme is blind if $\operatorname{Adv}_{\mathrm{BS}, \mathcal{A}}^{B \operatorname{lind}}(\lambda)$ is a negligible function of $\lambda$ for any polynomial time adversary $\mathcal{A}$.

To define unforgeability, we consider an adversary $\mathcal{A}$, having oracle access to the function $\operatorname{Issue}_{\mathrm{BS}}\left(\cdot, \mathrm{sk}_{\mathrm{BS}}\right)$, for adversarially chosen first parameter, running in an experiment as in Figure 2. We define $\operatorname{Adv}_{\mathrm{BS}, \mathcal{A}}^{\text {Unforge }}(\lambda)=$ $\operatorname{Pr}\left[\operatorname{Exp}_{\mathrm{BS}, \mathcal{A}}^{\text {Unforge }}(\lambda)=1\right]$ and we say that the scheme is unforgeable if $\operatorname{Adv}_{\mathrm{BS}, \mathcal{A}}^{\text {Unforge }}(\lambda)$ is a negligible function of $\lambda$ for any polynomial time adversary $\mathcal{A}$.

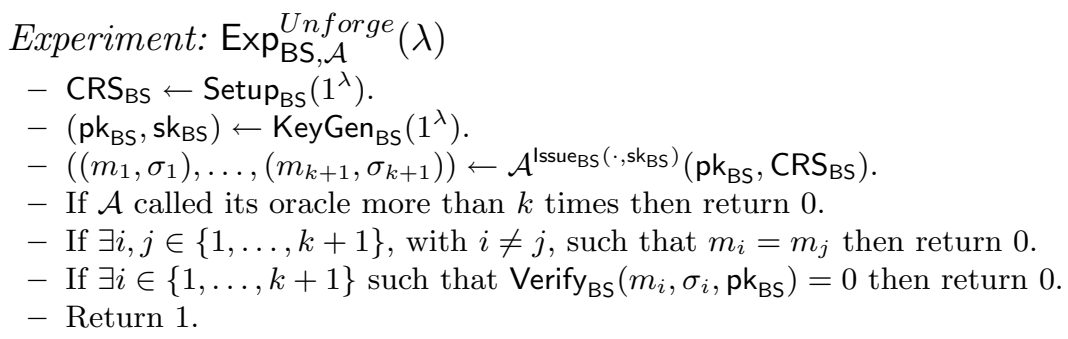

Fig. 2. The Unforgeability experiment

Note that our definition of forgery is not that of strong unforgeability; we do not require the adversary to not be able to output a new signature on an old message. This is because the final signature in our scheme will be a CL signature, which is always randomizable. Hence, our blind 
signature scheme may not be suitable in applications which require the strong unforgeability definition.

\section{Bilinear Groups and Associated Hard Problems}

In this section, we introduce the basic mathematical constructs needed to present our scheme. Namely abelian groups admitting a bilinear pairing and the associated hard problems.

Bilinear Groups: Bilinear groups are a set of three groups $\mathbb{G}_{1}, \mathbb{G}_{2}$ and $\mathbb{G}_{T}$, of prime order $p$, along with a bilinear map (a deterministic function) $\hat{t}$ which takes as input one element in $\mathbb{G}_{1}$ and one element in $\mathbb{G}_{2}$ and outputs an element in $\mathbb{G}_{T}$. We shall write $\mathbb{G}_{1}$ and $\mathbb{G}_{2}$ additively (with identity element 0 ), and $\mathbb{G}_{T}$ multiplicatively (with identity element 1 ), and write $\mathbb{G}_{1}=\left\langle P_{1}\right\rangle, \mathbb{G}_{2}=\left\langle P_{2}\right\rangle$, for two explicitly given generators $P_{1}$ and $P_{2}$. Multiplication by an integer $x$ in the group $\mathbb{G}_{1}$ (resp. $\mathbb{G}_{2}$ ) will be denote by $[x] P_{1}$ (resp. $\left.[x] P_{2}\right)$. We define $\mathcal{P}=\left(p, \mathbb{G}_{1}, \mathbb{G}_{2}, \mathbb{G}_{T}, \hat{t}, P_{1}, P_{2}\right)$ to be the set of pairing group parameters.

The function $\hat{t}$ must have the following three properties:

1. Bilinearity: $\forall Q_{1} \in \mathbb{G}_{1}, \forall Q_{2} \in \mathbb{G}_{2}, \forall x, y \in \mathbb{Z}$, we have

$$
\hat{t}\left([x] Q_{1},[y] Q_{2}\right)=\hat{t}\left(Q_{1}, Q_{2}\right)^{x y} .
$$

2. Non-Degeneracy: The value $\hat{t}\left(P_{1}, P_{2}\right)$ generates $\mathbb{G}_{T}$.

3. The function $\hat{t}$ is efficiently computable.

In practice, there are a number of different types of bilinear groups one can take, each giving rise to different algorithmic properties and different hard problems. Following [15] we categorize pairings into three distinct types (other types are possible, but the following three are the main ones utilized in practical protocols).

- Type-1: This is the symmetric pairing setting in which $\mathbb{G}_{1}=\mathbb{G}_{2}$.

- Type-2: Here we have $\mathbb{G}_{1} \neq \mathbb{G}_{2}$, but there is an efficiently computable isomorphism $\psi: \mathbb{G}_{2} \longrightarrow \mathbb{G}_{1}$ where $\psi\left(P_{2}\right)=P_{1}$.

- Type-3: Again $\mathbb{G}_{1} \neq \mathbb{G}_{2}$, but now there is no known efficiently computable isomorphism.

In this paper, we shall always consider Type-3 pairings. Such pairings can be efficiently realised; by taking $\mathbb{G}_{1}$ to be the set of points of order $p$ of an elliptic curve over $\mathbb{F}_{q}$ with a "small" embedding degree $k$; by taking $\mathbb{G}_{2}$ to be the set of points of order $p$ on a twist of the same elliptic curve 
over $\mathbb{F}_{q^{e}}$, for some divisor $e$ of $k$; and $\mathbb{G}_{T}$ to be the subgroup of order $p$ in the finite field $\mathbb{F}_{q^{k}}$.

For a security parameter $\lambda$, we let $\operatorname{Setup}_{\mathrm{Grp}}\left(1^{\lambda}\right)$ denote an algorithm which produces a pairing group instance $\mathcal{P}$ of Type-3.

HARD PROBLEMS: The security of our scheme rests on a variant of the following assumption, introduced in [22] in the case of Type-1 pairings; we present this problem in it generality for all pairings,

Definition 1 (LRSW Assumption). If $\mathcal{A}$ is an algorithm which is given access to an oracle $\mathcal{O}_{[x] P_{2},[y] P_{2}}(\cdot)$ that on input of $m \in \mathbb{F}_{p}$ outputs $(A, B, C)=(A,[y] A,[x+m \cdot x \cdot y] A)$, for some random $A \in \mathbb{G}_{1} \backslash\{0\}$, we let $Q$ denote the set of queries made by $\mathcal{A}$ to $\mathcal{O}_{[x] P_{2},[y] P_{2}}(\cdot)$.

The LRSW assumption is said to hold for the output of Setup $\mathrm{Grp}_{\text {if }}$ for all probabilistic polynomial time adversaries $\mathcal{A}$, and all outputs of Setup $_{G r p}$, the following probability is negligible in the security parameter $\lambda$,

$$
\begin{aligned}
\operatorname{Pr} & {\left[x \leftarrow \mathbb{F}_{p}, y \leftarrow \mathbb{F}_{p}, X \leftarrow[x] P_{2}, Y \leftarrow[y] P_{2},\right.} \\
& (Q, m, A, B, C) \leftarrow \mathcal{A}^{\mathcal{O}_{X, Y}(\cdot)}(\mathcal{P}, X, Y): \\
& m \notin Q \wedge m \in \mathbb{F}_{p} \backslash\{0\} \wedge \\
& \left.A \in \mathbb{G}_{1} \backslash\{0\} \wedge B=[y] A \wedge C=[x+m \cdot x \cdot y] A\right]
\end{aligned}
$$

In [22], it was shown that the LRSW assumption holds in the GGM and is independent of the DDH assumption. The LRSW assumption is the underlying hard problem behind the Camenisch-Lysyanskaya (CL) signature scheme.

Definition 2 (Camenisch-Lysyanskaya Signature Scheme). The $C L$ signature scheme is given by the following triple of algorithms given an output $\mathcal{P}$ of $\operatorname{Setup}_{\mathrm{Grp}}\left(1^{\lambda}\right)$.

- $\operatorname{KeyGen}(\mathcal{P}):$ Set sk $C L \leftarrow(x, y) \in \mathbb{F}_{p}^{2}$ and $\mathrm{pk}_{C L} \leftarrow(X, Y)=\left([x] P_{2},[y] P_{2}\right)$.

- $\operatorname{Sign}\left(m, \mathrm{sk}_{C L}\right):$ Select $A \leftarrow \mathbb{G}_{1} \backslash\{0\}$, and then set $B \leftarrow[y] A, C=$ $[x+m \cdot x \cdot y]$. Output $(A, B, C)$.

- $\operatorname{Verify}\left(m,(A, B, C), \mathrm{pk}_{C L}\right):$ Output true if and only if $\hat{t}\left(B, P_{2}\right)=\hat{t}(A, Y)$ and $\hat{t}\left(C, P_{2}\right)=\hat{t}(A, X) \cdot \hat{t}(B, X)^{m}$.

Indeed, the LRSW problem and the EF-CMA security of the CL signature scheme are equivalent since the oracle in the LRSW assumption produces CL signatures, and the output of the adversary against the LRSW assumption corresponds precisely to a forger who can construct 
one more CL signature. This is mirrored below in the relationship between the hardness of our E-LRSW assumption and the unforgeability of our scheme.

We shall require a strengthening of the LRSW assumption, a variant of which was first proposed by Chen et. al [12]. We first present the strengthening of Chen et. al, called the Blind-LRSW assumption, and then we present our modification which we call the Extended-LRSW assumption (E-LRSW).

In the B-LRSW problem the oracle provided to the adversary does not take as input an element $m \in \mathbb{F}_{p}$, but instead takes as input an element $M \in \mathbb{G}_{1}$. The output of the oracle is a CL signature on the discrete logarithm $m$ of $M$ with respect to $P_{1}$. The output of the adversary against this modified LRSW assumption is still a valid CL signature on a new element $m$. Following [12] we call this the Blind-LRSW (B-LRSW) assumption, since the adversary is given access to an oracle which can produce what are in effect blinded signatures. Formally we define

Definition 3 (B-LRSW Assumption). If $\mathcal{A}$ is an algorithm which is given access to an oracle, denoted by $\mathcal{O}_{[x] P_{2},[y] P_{2}}^{B}(\cdot)$, that on input of $M=[m] P_{1} \in \mathbb{G}_{1}$ outputs $(A, B, C)=(A,[y] A,[x+m \cdot x \cdot y] A)$, for some random $A \in \mathbb{G}_{1} \backslash\{0\}$, we let $Q$ denote the set of queries made by $\mathcal{A}$ to $\mathcal{O}_{[x] P_{2},[y] P_{2}}^{B}(\cdot)$.

The B-LRSW assumption is said to hold for the output of Setup Grp $_{1}$ if for all probabilistic polynomial time adversaries $\mathcal{A}$, and all outputs of Setup $_{\mathrm{Grp}}$, the following probability is negligible in the security parameter $\lambda$,

$$
\begin{aligned}
\operatorname{Pr} & {\left[x \leftarrow \mathbb{F}_{p}, y \leftarrow \mathbb{F}_{p}, X \leftarrow[x] P_{2}, Y \leftarrow[y] P_{2},\right.} \\
& (Q, m, A, B, C) \leftarrow \mathcal{A}^{\mathcal{O}_{X, Y}^{B}(\cdot)}(\mathcal{P}, X, Y): \\
& {[m] P_{1} \notin Q \wedge m \in \mathbb{F}_{p} \backslash\{0\} \wedge } \\
& \left.A \in \mathbb{G}_{1} \backslash\{0\} \wedge B=[y] A \wedge C=[x+m \cdot x \cdot y] A\right]
\end{aligned}
$$

Note that the oracle in the B-LRSW assumption, given access to $x$ and $y$, can compute its response by choosing $a \in \mathbb{F}_{p}$ at random and outputing the triple $\left([a] P_{1},[a \cdot y] P_{1},[a \cdot x] P_{1}+[a \cdot x \cdot y] M\right)$, i.e. the oracle does not need to be able to solve discrete logarithms if it has access to $x$ and $y$.

Also note, since an oracle query to $\mathcal{O}_{X, Y}(\cdot)$ can be simulated with an oracle query to $\mathcal{O}_{X, Y}^{B}(\cdot)$, and the output of the adversaries in the two problems is essentially identical, that an adversary $\mathcal{A}^{B}$ against the LRSW assumption can be turned into an adversary against the B-LRSW as- 
sumption (but it appears not vice-versa). Thus, the B-LRSW assumption is stronger than the LRSW assumption.

In our hard problem, shown to hold in the GGM in Appendix A, we extend the oracle in the B-LRSW assumption to provide some additional data about the values $A, x$ and $y$, with respect to a new public key element $Z=[z] P_{1}$. The output of the adversary still being a CamenischLysyanskaya signature.

Definition 4 (E-LRSW Assumption). If $\mathcal{A}$ is an algorithm which is given access to an oracle, denoted by $\mathcal{O}_{[x] P_{2},[y] P_{2},[z] P_{1}}^{E}(\cdot)$, that on input of $M=[m] P_{1} \in \mathbb{G}_{1}$ (for some unknown value of $m$ ) outputs $(A, B, C, D)=$ $(A,[y] A,[x+m \cdot x \cdot y] A,[x \cdot y \cdot z] A)$, for some random $A \in \mathbb{G}_{1} \backslash\{0\}$, we let $q$ denote the number of queries made by $\mathcal{A}$ to $\mathcal{O}_{[x] P_{2},[y] P_{2},[z] P_{1}}^{E}(\cdot)$.

The E-LRSW assumption is said to hold for the output of Setup $\mathrm{Grp}_{\text {, }}$, if for all probabilistic polynomial time adversaries $\mathcal{A}$, and all outputs of Setup $_{\mathrm{Grp}}$, the following probability is negligible in the security parameter $\lambda$,

$$
\begin{aligned}
\operatorname{Adv}_{\mathrm{E}-\mathrm{LRSW}, \mathcal{A}}(\lambda)=\operatorname{Pr} & x \leftarrow \mathbb{F}_{p}, y \leftarrow \mathbb{F}_{p}, z \leftarrow \mathbb{F}_{p}, \\
& X \leftarrow[x] P_{2}, Y \leftarrow[y] P_{2}, Z \leftarrow[z] P_{1} \\
& \left(\left\{m_{i}, A_{i}, B_{i}, C_{i}\right\}_{i=1}^{q+1}\right) \leftarrow \mathcal{A}^{\mathcal{O}_{X, Y, Z}^{E}(\cdot)}(\mathcal{P}, X, Y, Z): \\
& \text { For all } 1 \leq i \leq q+1 \text { we have } m_{i} \in \mathbb{F}_{p} \backslash\{0\} \wedge \\
& A_{i} \in \mathbb{G}_{1} \backslash\{0\} \wedge B_{i}=[y] A_{i} \wedge C_{i}=\left[x+m_{i} \cdot x \cdot y\right] A_{i} \\
& \left.\wedge\left(\text { if } i \neq j \text { then } m_{i} \neq m_{j}\right)\right]
\end{aligned}
$$

Note we present this assumption in terms of a one-more problem rather than in the form of the B-LRSW assumption. This is because the extra item $D$ allows the adversary to "open" the signature in one of a number of ways, if he can recover $z$ by solving the underlying discrete logarithm problem. However, the intuition is that he needs to commit to how he is going to open the signature before he sends the request to the oracle. We formalise this intuition when we present a proof in the GGM of the difficulty of the E-LRSW problem.

We note that our E-LRSW assumption is in some sense to the LRSW assumption, as the HSDH assumption from [8] is to the SDH assumption. In that the "queries" $\left(\left[1 /\left(x+c_{i}\right)\right] P_{1}, c_{i}\right)$ in the SDH assumption are replaced by "blinded queries" $\left(\left[1 /\left(x+c_{i}\right)\right] P_{1},\left[c_{i}\right] Q_{1},\left[c_{i}\right] P_{2}\right)$ in the HSDH assumption, and the output value $\left(\left[1 /\left(x+c^{*}\right)\right] P_{1}, c^{*}\right)$ in the SDH assumption is replaced by the blinded output value $\left(\left[1 /\left(x+c^{*}\right)\right] P_{1},\left[c^{*}\right] Q_{1},\left[c^{*}\right] P_{2}\right)$ in the HSDH assumption, where $P_{1} \in \mathbb{G}_{1}, P_{2} \in \mathbb{G}_{2}, Q_{1} \leftarrow \mathbb{G}_{1}, x, c_{i} \leftarrow \mathbb{Z}_{p}^{*}$ and some $c^{*} \in \mathbb{Z}_{p}^{*}$ where $c^{*} \notin\left\{c_{i}\right\}$. 
Recall the blind signature scheme below is akin to the blind-unblind schemes in the ROM, such as those based on RSA. It should therefore not be surprising that we need to strengthen the security assumption of the underling signature scheme so as to cope with blindness properties. This is exactly what is required for the ROM-based RSA and discrete logarithm based schemes $[3,5]$

\section{Our Scheme}

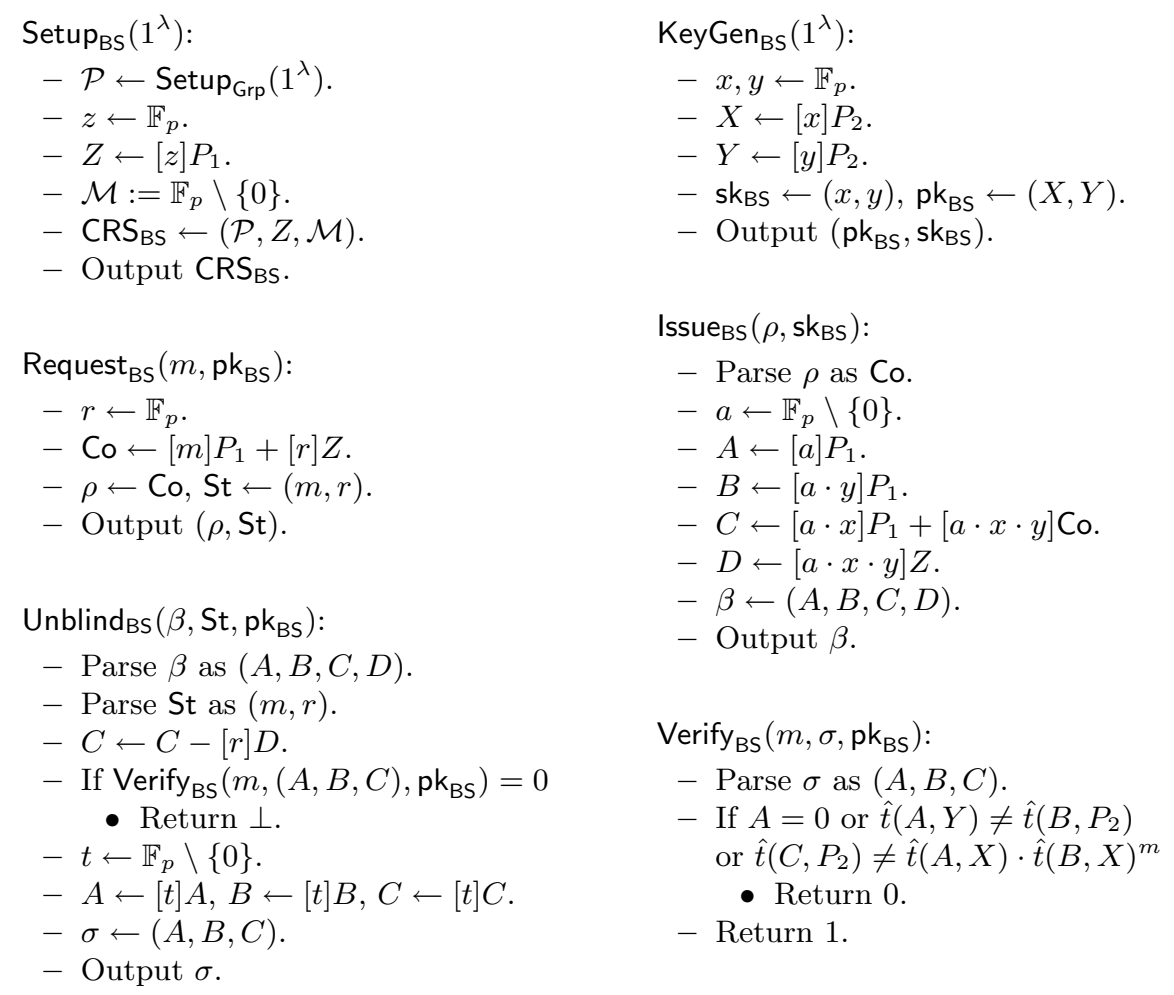

Fig. 3. Our blind signature scheme

In Figure 3, we define the algorithms which make up our blind signature scheme. Notice, that $\beta$ contains a CL signature on the "hidden" message $m+z \cdot r$, but no party knows the value of $z$. The signer is signing a message he does not know. Indeed, the user also does not know the message (unless he picks $r=0$ ), but he is able to unblind this signature to produce a valid CL signature on $m$ using his value $r$. To see 
that the unblinded signature is valid, notice that the value of $C$ (before multiplication by $t$ ) is equal to

$$
\begin{aligned}
C & =\left([a \cdot x] P_{1}+[a \cdot x \cdot y] \mathrm{Co}\right)-[r] D, \\
& =[x] A+[a \cdot x \cdot y] \cdot\left([m] P_{1}+[r] Z\right)-[r] D, \\
& =[x+m \cdot x \cdot y] A+[a \cdot x \cdot y \cdot r] Z-[r] D, \\
& =[x+m \cdot x \cdot y] A .
\end{aligned}
$$

Then notice, that even the revealed signature provides no linkage with the values signed, due to the fact that CL signatures for Type-3 pairings are unlinkable once randomized. In our security proof we will confirm this intuition.

\section{Proof of Security}

First we prove that the scheme is blind, then we show that it is unforgeable.

Theorem 1. The above blind signature scheme is perfectly blind. In particular, if $\mathcal{A}$ is an adversary against the blindness of the above blind signature scheme, then

$$
\operatorname{Adv}_{\mathrm{BS}, \mathcal{A}}^{\text {Blind }}(\lambda)=0 .
$$

Proof. We reduce blindness to the hiding property of Pedersen commitments [26], which is defined by the following experiment:

$$
\begin{aligned}
& \text { Experiment: } \operatorname{Exp}_{\text {Pedersen, } \mathcal{C}}^{\text {Hiding }}(\lambda) \\
& - \text { sk } \leftarrow \mathbb{F}_{p}, b \leftarrow\{0,1\}, r_{0}, r_{1} \leftarrow \mathbb{F}_{p} . \\
& - \text { pk } \leftarrow[\mathrm{sk}] P_{1} . \\
& -\left(m_{0}, m_{1}, \mathrm{St}\right) \leftarrow \mathcal{C}_{1}(\mathrm{pk}), \text { with } m_{i} \in \mathbb{F}_{p} . \\
& -C_{0} \leftarrow\left[m_{b}\right] P_{1}+\left[r_{0}\right] \mathrm{pk} . \\
& -C_{1} \leftarrow\left[m_{1-b}\right] P_{1}+\left[r_{1}\right] \mathrm{pk} . \\
& -b^{\prime} \leftarrow \mathcal{C}_{2}\left(C_{0}, C_{1}, \text { St }\right) . \\
& - \text { If } b=b^{\prime} \text { then return } 1, \text { else return } 0 .
\end{aligned}
$$

If $\mathcal{C}$ is an adversary in the above experiment then we define

$$
\operatorname{Adv}_{\text {Pedersen }, \mathcal{C}}^{\text {Hiding }}(\lambda)=\left|2 \cdot \operatorname{Pr}\left[\operatorname{Exp}_{\text {Pedersen }, \mathcal{C}}^{\text {Hiding }}(\lambda)=1\right]-1\right| .
$$

That Pedersen commitments are perfectly hiding is a classic result. Thus, we have $\operatorname{Adv}_{\text {Pedersen, } \mathcal{C}}^{\text {Hiding }}(\lambda)=0$, i.e. $\operatorname{Pr}\left[\operatorname{Exp}_{\text {Pedersen, } \mathcal{C}}^{\text {Hiding }}(\lambda)=1\right]=1 / 2$.

We now turn to the security game for blindness for our blind signature scheme. We let $\mathrm{G}_{0}$ denote the experiment played by the adversary $\mathcal{A}$ 
against the blindness property of the scheme, see Figure 4 . We let $E$ denote the event that the guess stage of the adversary is passed the pair $\left(\sigma_{0}, \sigma_{1}\right)=(\perp, \perp)$. We clearly have

$$
\begin{aligned}
\operatorname{Pr}\left[\operatorname{Exp}_{\mathrm{BS}, \mathcal{A}}^{B \operatorname{lind}}(\lambda)=1\right] \leq & \operatorname{Pr}[E] \cdot \operatorname{Pr}\left[\operatorname{Exp}_{\mathrm{BS}, \mathcal{A}}^{\text {Blind }}(\lambda)=1 \mid E\right] \\
& +\operatorname{Pr}[\neg E] \cdot \operatorname{Pr}\left[\operatorname{Exp}_{\mathrm{BS}, \mathcal{A}}^{\text {Blind }}(\lambda)=1 \mid \neg E\right] .
\end{aligned}
$$

It is clear that in the case that the event $E$ happens, then the adversary $\mathcal{A}$ can be turned into an adversary against the Pedersen commitments $\mathrm{Co}_{0}$ and $\mathrm{Co}_{1}$. We hence have that

$$
\operatorname{Pr}\left[\operatorname{Exp}_{\mathrm{BS}, \mathcal{A}}^{\text {Blind }}(\lambda)=1 \mid E\right]=\operatorname{Pr}\left[\operatorname{Exp}_{\text {Pedersen }, \mathcal{C}}^{\text {Hiding }}(\lambda)\right]=1 / 2 .
$$

In the following, we will show that we also have

$$
\operatorname{Pr}\left[\operatorname{Exp}_{\mathrm{BS}, \mathcal{A}}^{\text {Blind }}(\lambda)=1 \mid \neg E\right]=\operatorname{Pr}\left[\operatorname{Exp}_{\text {Pedersen }, \mathcal{C}}^{\text {Hiding }}(\lambda)\right]=1 / 2,
$$

and so

$$
\operatorname{Pr}\left[\operatorname{Exp}_{\mathrm{BS}, \mathcal{A}}^{\text {Blind }}(\lambda)=1\right] \leq(\operatorname{Pr}[E]+\operatorname{Pr}[\neg E]) / 2=1 / 2,
$$

from which our result will follow.

So from now on assume that the event $\neg E$ happens. In which case there is no need for the challenger to check the returned pre-signatures are valid. Thus, the challenger can obtain valid signatures by ignoring the values returned by the adversary and simply generating the signatures himself; since he knows the secret key. Hence, from game $G_{0}$ we can make a hop to game $\mathrm{G}_{1}$, also presented in Figure 4.

If we let $\mathcal{S}_{0}(m)$ denote the distribution of signatures returned to the final stage of the adversary in game $\mathrm{G}_{0}$ on message $m$ assuming event $\neg E$ happens, and $\mathcal{S}_{1}(m)$ the distribution in game $\mathrm{G}_{1}$, we see that $\mathcal{S}_{0}(m)$ is identically distributed to $\mathcal{S}_{1}(m)$. This is because in $\mathrm{G}_{0}$ we randomize a specific CL signature, whereas in $G_{1}$ we produce a new independent CL signature. It is easy to see that these two operations have the same effect on the distribution of the final signature passed to the final stage of the adversary. If we let $\operatorname{Pr}\left[\mathrm{G}_{i}\right]$ denote the probability of the adversary winning in game $\mathrm{G}_{i}$, we have $\operatorname{Pr}\left[\mathrm{G}_{0} \mid \neg E\right]=\operatorname{Pr}\left[\mathrm{G}_{1}\right]$.

We now show that an adversary playing $G_{1}$, must have zero advantage. To see this note that the value Co returned by Request ${ }_{B S}$ is a Pedersen commitment to the value $m$, hence an adversary in game $\mathrm{G}_{1}$ can be turned into an adversary against the hiding property of Pedersen commitments. That we have $\operatorname{Pr}\left[G_{1}\right]=\operatorname{Pr}\left[\operatorname{Exp}_{\text {Pedersen } \mathcal{C}}^{\text {Hiding }}(\lambda)\right]$ follows immediately; we simply translate the $\left(m_{0}, m_{1}\right)$ output by the first stage of adversary $\mathcal{A}$ in game 


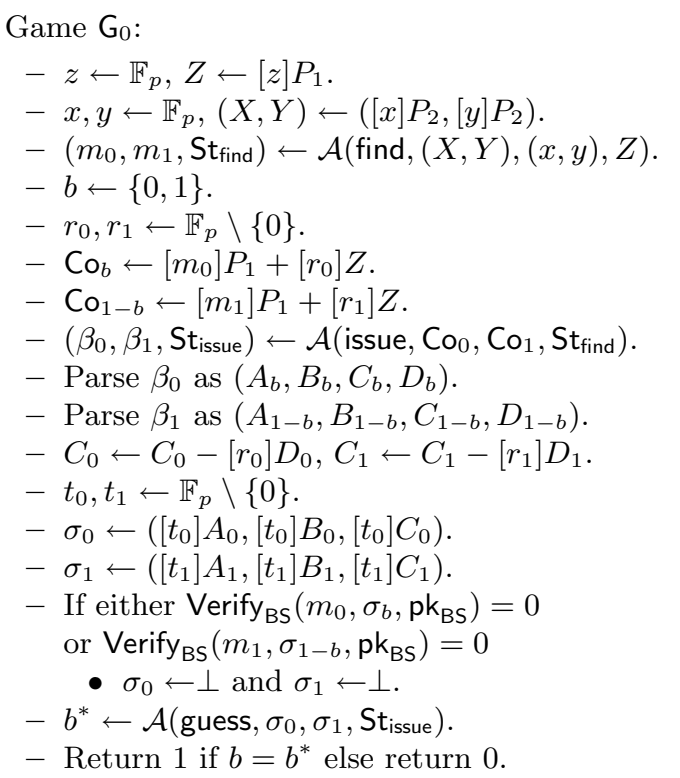

Game $\mathrm{G}_{1}$ :

$-z \leftarrow \mathbb{F}_{p}, Z \leftarrow[z] P_{1}$.

$-x, y \leftarrow \mathbb{F}_{p},(X, Y) \leftarrow\left([x] P_{2},[y] P_{2}\right)$.

$-\left(m_{0}, m_{1}, \mathrm{St}_{\text {find }}\right) \leftarrow \mathcal{A}($ find $,(X, Y),(x, y), Z)$.

$-b \leftarrow\{0,1\}$.

$-r_{0}, r_{1} \leftarrow \mathbb{F}_{p} \backslash\{0\}$.

$-\mathrm{Co}_{b} \leftarrow\left[m_{0}\right] P_{1}+\left[r_{0}\right] Z$.

$-\mathrm{Co}_{1-b} \leftarrow\left[m_{1}\right] P_{1}+\left[r_{1}\right] Z$.

$-\left(\beta_{0}, \beta_{1}, \mathrm{St}_{\text {issue }}\right) \leftarrow \mathcal{A}$ (issue, $\left.\mathrm{Co}_{0}, \mathrm{Co}_{1}, \mathrm{St}_{\text {find }}\right)$.

$-a_{0}, a_{1} \leftarrow \mathbb{F}_{p} \backslash\{0\}$.

- $\sigma_{0} \leftarrow\left(\left[a_{0}\right] P_{1},\left[a_{0} \cdot y\right] P_{1},\left[a_{0} \cdot x+a_{0} \cdot x \cdot y \cdot m_{0}\right] P_{1}\right)$.

$-\sigma_{1} \leftarrow\left(\left[a_{1}\right] P_{1},\left[a_{1} \cdot y\right] P_{1},\left[a_{1} \cdot x+a_{1} \cdot x \cdot y \cdot m_{1}\right] P_{1}\right)$.

$-b^{*} \leftarrow \mathcal{A}$ (guess, $\left.\sigma_{0}, \sigma_{1}, \mathrm{St}_{\text {issue }}\right)$.

- Return 1 if $b=b^{*}$ else return 0 .

Fig. 4. The two games $G_{0}$ and $G_{1}$.

$\mathrm{G}_{1}$, into the challenge for the Pedersen hiding game; this provides the input into the second stage of adversary $\mathcal{A}$; the input to the third stage of adversary $\mathcal{A}$ is then independent of the values returned by our first game hop.

Note that the above proof holds even if the adversary can select the value of the secret key $\mathbf{s k}_{\mathrm{BS}}$, as long as the challenger is able to extract it so as to answer the queries for game $G_{1}$.

We now turn to show that our scheme is unforgeable.

Theorem 2. If the E-LRSW assumption holds then the above blind signature scheme is unforgeable. In particular, if $\mathcal{A}$ is an adversary against the unforgeability of the above blind signature scheme, then there is an adversary $\mathcal{B}$ which solves the $E-L R S W$ problem such that

$$
\operatorname{Adv}_{\mathrm{BS}, \mathcal{A}}^{\text {Unforge }}(\lambda)=\operatorname{Adv}_{\mathrm{E}-\mathrm{LRSW}, \mathcal{B}}(\lambda) .
$$

Proof. Let $\mathcal{A}$ denote an adversary against the unforgeability experiment of the blind signature scheme. We shall use $\mathcal{A}$ to construct an algorithm which solves the E-LRSW problem. Let $(\mathcal{P}, X, Y, Z)$ denote the input to the E-LRSW problem instance. Algorithm $\mathcal{B}$ sets up the CRS and the 
public keys for the blind signature scheme by setting $\mathrm{CRS}_{\mathrm{BS}} \leftarrow\left(\mathcal{P}, Z, \mathbb{F}_{p} \backslash\right.$ $\{0\})$ and $\mathrm{pk}_{\mathrm{BS}} \leftarrow(X, Y)$.

Algorithm $\mathcal{B}$ now calls adversary $\mathcal{A}$. At some point $\mathcal{A}$ will make one

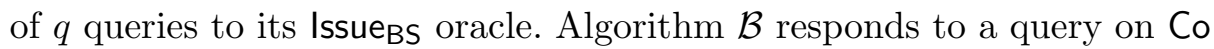
as follows: It passes the value Co to its E-LRSW oracle $\mathcal{O}_{X, Y, Z}^{E}$ so as to obtain a tuple

$$
(A, B, C, D)=(A,[y] A,[x+t \cdot x \cdot y] A,[x \cdot y \cdot z] A),
$$

where $\mathrm{Co}_{\mathrm{o}}=[t] P_{1}$. Notice that if $t=m+r \cdot z$ then $(A, B, C, D)$ is a valid response for the commitment to the message $m$. The tuple $(A, B, C, D)$ is now passed back to algorithm $\mathcal{A}$.

Eventually, $\mathcal{A}$ will terminate by outputting a set of $q+1$ tuples $\left(m_{i}, A_{i}, B_{i}, C_{i}\right)$ where $\left(A_{i}, B_{i}, C_{i}\right)$ is a valid CL signature on $m_{i}$. By returning this list to its challenger, the adversary thereby solves the ELRSW assumption.

Note that if we have modified the protocol such that the signer appends a NIZK proof of correctness of its response, then the above proof can be applied in the case where the adversary generates his own secret keys; as long as one adds an additional game-hop to enable the extraction of the witness underlying the NIZK proof system. This is a standard technique so we leave it to the reader.

\section{Acknowledgements}

The authors would like to thank the EU projects CACE, eCrypt-2 and the ERC Advanced Grant to Nigel Smart for funding the work in this paper. The second author was supported by the EPSRC under grant EP/H043454/1, and was also partially funded by a Royal Society Wolfson Merit Award, and Google Inc. The authors would like to thank Bogdan Warinschi for useful conversations whilst the work was carried out.

\section{References}

1. M. Abe. A secure three-move blind signature scheme for polynomially many signatures. In Advances in Cryptology - Eurocrypt 2001, Springer LNCS 2045 , 136-151, 2001.

2. M. Abe, G. Fuchsbauer, J. Groth, K. Haralambiev and M. Ohkubo. Structurepreserving signatures and commitments to group elements. In Advances in Cryptology - Crypto 2010, Springer LNCS 6223, 209-236, 2010. 
3. M. Bellare, C. Namprempre, D. Pointcheval, and M. Semanko. The one-more-RSAinversion problems and the security of Chaum's blind signature scheme. Journal of Cryptology, 16(3), 185-215, 2003.

4. M. Bellare and P. Rogaway. The exact security of digital signatures - How to sign with RSA and Rabin. In Advances in Cryptology - Eurocrypt '96, Springer LNCS 1070, 399-416, 1996.

5. A. Boldyreva. Efficient threshold signatures, multisignatures and blind signatures based on the Gap-Diffie-Hellman-Group signature scheme. In Public Key Cryptography - PKC 2003, Springer LNCS 2567, 31-46, 2003.

6. D. Boneh and X. Boyen. Short signatures without random oracles. Journal of Cryptology, 21(2), 149-177, 2008.

7. D. Boneh, B. Lynn and H. Shacham. Short signatures from the Weil pairing. Journal of Cryptology, 17(4), 297-319, 2004.

8. X. Boyen and B. Waters. Full-domain subgroup hiding and constant-size group signatures. In Public Key Cryptography - PKC 2007, Springer LNCS 4450, 1-15, 2007.

9. J. Camensich, M. Koprowski and B. Warinschi. Efficient blind signatures without random oracles. In Security in Communication Networks - SCN 2004, Springer LNCS 3352, 134-148, 2004.

10. J. Camenisch and A. Lysyanskaya. Signature schemes and anonymous credentials from bilinear maps. In Advances in Cryptology - CRYPTO 2004, Springer LNCS 3152, 56-72, 2004.

11. D. Chaum. Blind signatures for untraceable payments. In Advances in Cryptology - CRYPTO 1982, Plenum Press, 199-203, 1983.

12. L. Chen, P. Morrissey and N.P. Smart. DAA: Fixing the pairing based protocols. IACR e-print 2009/198. http://eprint.iacr.org/2009/198.

13. M. Fischlin. Round-optimal composable blind signatures in the common reference string model. In Advances in Cryptology - CRYPTO 2006, Springer LNCS 4117, 60-77, 2006.

14. G. Fuchsbauer. Automorphic signatures in bilinear groups and an application to round-optimal blind signatures. IACR e-print 2009/320. http://eprint.iacr. org/2009/320.

15. S. Galbraith, K. Paterson and N.P. Smart. Pairings for cryptographers. Discrete Applied Mathematics, 156, 3113-3121, 2008

16. S. Garg, V. Rao, A. Sahai, D. Schröder and D. Unruh. Round optimal blind signatures. In Advances in Cryptology - CRYPTO 2011, Springer LNCS 6841, 630-648, 2011.

17. J. Groth and A. Sahai. Efficient non-interactive proof systems for bilinear groups. In Advances in Cryptology - EUROCRYPT 2008, Springer LNCS 4965, 415-432, 2008.

18. J. Groth and A. Sahai. Efficient non-interactive proof systems for bilinear groups (full version). http://www.brics.dk/ jg/WImoduleFull.pdf

19. T. Jager and A. Rupp. The semi-generic group model and applications to pairingbased cryptography. In Advances in Cryptology - ASIACRYPT 2010, Springer LNCS 6477, 539-556, 2010.

20. A. Juels, M. Luby and R. Ostrovsky. Security of blind digital signatures. In Advances in Cryptology - CRYPTO '97, Springer LNCS 1294, 150-164, 1997.

21. A. Kiayias and H.-S. Zhou. Concurrent blind signatures without random oracles. In Security and Cryptography for Networks - SCN 2006, Springer LNCS 4116, 49-62, 2006. 
22. A. Lysyanskaya, R. Rivest, A. Sahai and S. Wolf. Pseudonym systems. In Selected Areas in Cryptography - SAC 1999, Springer LNCS 1758, 184-199, 1999.

23. U. Maurer. Abstract models of computation in cryptography. In Cryptography and Coding 2005, Springer LNCS 3796, 1-12, 2005.

24. M. Naor. On cryptographic assumptions and challenges. In Advances in Cryptology - Crypto 2003, Springer LNCS 2729, 96-109, 2003.

25. T. Okamoto. Efficient blind and partially blind signatures without random oracles. In Theory of Cryptography Conference - TCC 2006, Springer LNCS 3876, 80-99, 2006.

26. T.P. Pedersen. Non-interactive and information-theoretic secure verifiable secret sharing. In Advances in Cryptology - CRYPTO '91, Springer LNCS 576, 129-140, 1991.

27. D. Pointcheval and J. Stern. Security arguments for digital signatures and blind signatures. Journal of Cryptology, 13(3), 361-396, 2000.

28. J. T. Schwartz. Fast Probabilistic Algorithms for Verification of Polynomial Identities. In J. $A C M, \mathbf{2 7}, 701-717,1980$.

29. V. Shoup. Lower bounds for discrete logarithms and related problems. In Advances in Cryptology - Eurocrypt 1997, Springer LNCS 1233, 256-266, 1997.

\section{A E-LRSW in the Generic Group Model}

We now show that the E-LRSW assumption holds in the GGM. Whilst not providing complete evidence that a given problem is hard, a GGM proof can be used to gain confidence in an underlying assumption. For more details of the GGM and its application to other problems, see [29, 23 . One could also examine the problem in terms of the semi-generic group model of [19], however we feel this would not add anything as the problem in $\mathbb{G}_{T}$ one would reduce security to would be almost identical to the initial input problem.

Our model used below models the groups $\mathbb{G}_{1}, \mathbb{G}_{2}$ and $\mathbb{G}_{T}$ as generic groups, and in addition gives the adversary access to an oracle which simulates the pairing operation.

Theorem 3. Let $\mathcal{A}$ denote an adversary in the generic group model against the E-LRSW assumption. Assume $\mathcal{A}$ makes $q_{G}$ queries to the group operations, $q_{P}$ queries to the pairing and $q_{O}$ queries to the E-LRSW oracle $\mathcal{O}^{E}$. If we set $n=5+q_{G}+4 q_{O}+q_{P}$ then the probability of the adversary winning the $E-L R S W$ game is $O\left(n^{2} \cdot q_{O} / p\right)$, where $p$ is the (prime) order of the generic group.

Proof. A GGM adversary against E-LRSW works by interacting with the group operations, pairing and $\mathcal{O}^{E}$ oracles via group handles. We first present how the adversary uses these handles to provide access to these operations. Different authors use slightly different notions as to how this 
is done, but all are essentially equivalent. The challenger keeps three lists $\mathcal{G}_{1}, \mathcal{G}_{2}, \mathcal{G}_{T}$ of pairs $(\sigma, P)$ where $\sigma$ is a "random" encoding of the group element chosen from some set $\mathcal{S}$ with $\# \mathcal{S}>3 \cdot p$, and $P$ is some polynomial in $\mathbb{F}_{p}\left[X, Y, Z, A_{1}, \ldots, A_{q_{O}}\right]$. We take the simplified GGM model in which adversaries are unable to generate completely random elements independently, i.e. new elements can only be produced using the algorithms below. This is purely for exposition, one can modify our following argument to cope with this additional ability, but at the expense of introducing an increase in the number of indeterminants.

To each list we associate an Update operation, that takes as input the specific list $\mathcal{G}$, and a polynomial $P$. The function Update $(\mathcal{G}, P)$ searches the list for a pair whose second component is equal to $P$, if it finds one then it returns the first component as a result. Otherwise a new element $\sigma$ is pulled from $\mathcal{S}$, distinct from all other elements in $\mathcal{S}$ used so far, and the pair $(\sigma, \mathcal{P})$ is added to the list. The value $\sigma$ is then returned. The values $\sigma$ are the handles used by the adversary to represent the group elements; note the adversary at no point gets access to the second element in the pairs.

The lists are first initialized by executing the following five operations:

$$
\begin{aligned}
& \operatorname{Update}\left(\mathcal{G}_{1}, 1\right), \quad \operatorname{Update}\left(\mathcal{G}_{1}, Z\right), \\
& \operatorname{Update}\left(\mathcal{G}_{2}, 1\right), \quad \operatorname{Update}\left(\mathcal{G}_{2}, X\right), \quad \operatorname{Update}\left(\mathcal{G}_{2}, Y\right) .
\end{aligned}
$$

The adversary now interacts with these lists via the following operations. Group Operations: The adversary has access to three oracles $\mathcal{O}_{1}, \mathcal{O}_{2}, \mathcal{O}_{T}$ which allow him access to the group operations, via a subtraction operation. On a call to $\mathcal{O}_{i}\left(\sigma_{1}, \sigma_{2}\right)$ the challenger searches list $\mathcal{G}_{i}$ for pairs of the form $\left(\sigma_{1}, P_{1}\right)$ and $\left(\sigma_{2}, P_{2}\right)$. If two such pairs do not exist then the challenger returns $\perp$. Otherwise, the challenger returns to the adversary the output of Update $\left(\mathcal{G}_{i}, P_{1}-P_{2}\right)$. Given a subtraction operation we can define the identity $\mathcal{O}_{\mathcal{G}_{i}}$ of the group via $\mathcal{O}_{i}(\sigma, \sigma)$, negation $-\sigma$ via $\mathcal{O}_{i}\left(\mathcal{O}_{\mathcal{G}_{i}}, \sigma\right)$, and hence addition via $\mathcal{O}_{i}\left(\sigma_{1},-\sigma_{2}\right)$.

PAIRING Operation: The adversary has access to an oracle $\mathcal{O}_{P}$. When called with $\mathcal{O}_{P}\left(\sigma_{1}, \sigma_{2}\right)$ the challenger searches the list $\mathcal{G}_{1}$ for a pair of the form $\left(\sigma_{1}, P_{1}\right)$, and the list $\mathcal{G}_{2}$ for a pair of the form $\left(\sigma_{2}, P_{2}\right)$. Again, if no such pairs are found to exist then the challenger returns $\perp$. Otherwise, the challenger returns to the adversary the output of $\operatorname{Update}\left(\mathcal{G}_{T}, P_{1} \cdot P_{2}\right)$. E-LRSW ORACLE: The adversary may make up to $q_{O}$ queries of the oracle $\mathcal{O}^{E}(\sigma)$. We let $i$ denote the $i$ th such query. Upon receiving such a request, the challenger searches the $\mathcal{G}_{1}$ list for a pair $(\sigma, P)$. If no such pair 
exists then the challenger returns $\perp$. Otherwise, the challenger executes the operations, where $A_{i}, X, Y$ and $Z$ are the indeterminants introduced above,

$$
\begin{aligned}
\sigma_{A} & \leftarrow \operatorname{Update}\left(\mathcal{G}_{1}, A_{i}\right), \quad \sigma_{B} \leftarrow \operatorname{Update}\left(\mathcal{G}_{1}, A_{i} \cdot Y\right), \\
\sigma_{C} & \leftarrow \operatorname{Update}\left(\mathcal{G}_{1}, A_{i} \cdot X \cdot(1+Y \cdot P)\right), \quad \sigma_{D} \leftarrow \operatorname{Update}\left(\mathcal{G}_{1}, A_{i} \cdot X \cdot Y \cdot Z\right) .
\end{aligned}
$$

Returning the tuple $\left(\sigma_{A}, \sigma_{B}, \sigma_{C}, \sigma_{D}\right)$ to the adversary.

Using these oracles we can simulate the entire run of the adversary in the GGM, i.e. the adversary may make no decision which depends on the particular encoding of group elements used. Notice after executing these operations the total number of non-constant polynomials contained in the three lists $\mathcal{G}_{1}, \mathcal{G}_{2}$ and $\mathcal{G}_{T}$ is bounded from above by $m=5+q_{G}+4 q_{O}+q_{P}$.

We wish to show that the probability of such an adversary being successful is negligibly small. Thus, assume the adversary is successful in solving the E-LRSW problem. It will therefore eventually output a set of $q_{O}+1$ tuples

$$
\left\{m_{i}, \sigma_{A}^{(i)}, \sigma_{B}^{(i)}, \sigma_{C}^{(i)}\right\}_{i=1}^{q_{O}+1}
$$

where $m_{i} \in \mathbb{F}_{p} \backslash\{0\}$ are distinct and $\sigma_{A}^{(i)}, \sigma_{B}^{(i)}, \sigma_{C}^{(i)}$ are handles on group elements in $\mathbb{G}_{1}$. We let $P_{A}^{(i)}, P_{B}^{(i)}, P_{C}^{(i)}$ denote the associated polynomials related to these handles in the list $\mathcal{G}_{1}$.

As the output is supposed to correspond to a solution to the E-LRSW problem, these output polynomials can be assumed to satisfy, for some assignment $\left(x, y, z, a_{1}, \ldots, a_{q_{O}}\right) \in \mathbb{F}_{p}^{3+q_{O}}$ to the variables $\left(X, Y, Z, A_{1}, \ldots, A_{q_{O}}\right)$, the two equations

$$
\begin{aligned}
\left(Y \cdot P_{A}^{(i)}-P_{B}^{(i)}\right)\left(x, y, z, a_{1}, \ldots, a_{q_{O}}\right) & =0, \\
\left(X \cdot P_{A}^{(i)}+X \cdot m_{i} \cdot P_{B}^{(i)}-P_{C}^{(i)}\right)\left(x, y, z, a_{1}, \ldots, a_{q_{O}}\right) & =0 .
\end{aligned}
$$

From this we wish to derive a contradiction, i.e. conclude that the adversary cannot solve the E-LRSW assumption in the GGM.

Typically, a proof in the GGM proceeds by showing that the probability of an assignment to the variables resulting in either the output equations being satisfied, or two polynomials on the lists being equal at this assignment, is negligible. In most proofs it is obvious that the output polynomials do not result in the checking equations which are identically zero, however in our situation this is not obvious. In other words, it is not obvious that we do not have, for all $i$, that

$$
Y \cdot P_{A}^{(i)}-P_{B}^{(i)} \equiv X \cdot P_{A}^{(i)}+X \cdot m_{i} \cdot P_{B}^{(i)}-P_{C}^{(i)} \equiv 0 .
$$


Thus, we divide the proof into demonstrating three properties:

- Firstly, that the above set of equations is not identically zero. If this holds then we will be able to apply the standard Schwartz-Zippel lemma [28].

- Secondly, that the adversary does not learn, for a specific assignment to the variables, that it is not interacting with genuine group oracles. In other words, we apply the Schwartz-Zippel lemma to pairs of the resulting equations in the adversary's lists.

- Thirdly, that the chance of a specific assignment to the variables, satisfying the above set of (non-trivial) equalities is negligible. In this case, we apply the Schwartz-Zippel lemma to the output set of equations, having already determined by the first step that they are not identically zero.

Before examining these properties, we notice that the degree of the polynomials in $\mathcal{G}_{1}, \mathcal{G}_{2}$ and $\mathcal{G}_{T}$ can only be raised by application of the $\mathcal{O}_{P}$ and $\mathcal{O}^{E}$ oracles. Indeed, if $q_{O}$ is the total number of queries to $\mathcal{O}^{E}$ made by the adversary, then the total degree of all polynomials is bounded from above by $\mathrm{d}_{\max }=9 \cdot q_{O}+1$.

Property OnE: The first of these properties is often not addressed in GGM proofs since it is usually obvious that the required equation cannot hold for all assignments; consider, for example, the proof of difficulty of $\mathrm{CDH}$ in the GGM the output equation is a quadratic equation yet only linear operations on linear equations are allowed. Our situation is different, the oracle $\mathcal{O}^{E}$ allows us to obtain solutions which hold for all assignments.

We first observe that if we are trying to construct polynomials $P_{A}^{(i)}$ such that $P_{B}^{(i)}=Y \cdot P_{A}^{(i)}$, for polynomials $P_{A}^{(i)}, P_{B}^{(i)}$ in $\mathcal{G}_{1}$, then neither $P_{A}^{(i)}$ nor $P_{B}^{(i)}$ can involve any terms in $X$. This fact follows from the nature of the oracle queries available to the adversary. To see this we proceed by induction, assume no such polynomial $P_{A}^{(i)}$ exists which has positive degree in $X$ at the point where $\mathcal{A}$ makes its $j$ th query to the $\mathcal{O}^{E}$ oracle. Clearly, this property holds when $j=0$, so assume the property holds when at an arbitrary $j$. At the point of making the $(j+1)$-th query, the adversary has been able to produce linear combinations of the polyomials available before the $j$ th query, and those obtained from the $j$ th query. This is because only the $\mathcal{O}^{E}$ oracle allows the adversary to produce nonlinear operations on polynomials in the $\mathcal{G}_{1}$ list. But it is clear that the $j$ th query (followed by linear operations) cannot result in two polynomial 
with $\operatorname{deg}_{X}\left(P_{A}^{(i)}\right) \geq 1$ and $P_{B}^{(i)}=Y \cdot P_{A}^{(i)}$. Similarly we can conclude that $\operatorname{deg}_{Z}\left(P_{A}^{(i)}\right)=0$.

From this, we conclude that we must have

$$
P_{A}^{(i)}=\sum_{j=1}^{q} r_{i, j} \cdot A_{j} \text { and } P_{B}^{(i)}=\sum_{j=1}^{q} r_{i, j} \cdot Y \cdot A_{j}
$$

for some integer values $r_{i, j}$. From which it follows that

$$
P_{C}^{(i)}=\sum_{j=1}^{q} r_{i, j} \cdot A_{j} \cdot X \cdot\left(1+m_{i} \cdot Y\right)
$$

But the only way to produce a polynomial $P_{C}^{(i)}$ in the list $\mathcal{G}_{1}$, using our provided operations, which is divisible by a single power of $X$, would be to add together multiples of the polynomials associated with the $\sigma_{C}$ and $\sigma_{D}$ values output by the $\mathcal{O}^{E}$ oracle. In addition, the queries to this oracle would have been associated to polynomials of the form $f_{j}+g_{j} \cdot Z$, otherwise they could not then be used in constructing the output signatures. This means we have values $s_{i, j}, t_{i, j}, f_{j}, g_{j} \in \mathbb{Z}_{p}$ such that

$$
P_{C}^{(i)}=\sum_{j=1}^{q}\left(s_{i, j} \cdot A_{j} \cdot X \cdot\left(1+f_{j} \cdot Y+g_{j} \cdot Y \cdot Z\right)+t_{i, j} \cdot A_{j} \cdot X \cdot Y \cdot Z\right) .
$$

Equating coefficients in equations (1) and (2) we find that we must have

$$
r_{i, j}=s_{i, j}, \quad r_{i, j} \cdot m_{i}=s_{i, j} \cdot f_{j}, \text { and } 0=s_{i, j} \cdot g_{j}+t_{i, j},
$$

for all values $1 \leq i \leq q_{O}+1$ and $1 \leq j \leq q_{O}$. If, for any specific value of $i$, we have $s_{i, j}=0$ for all values of $j$, then the $i$ th output signature is of the form $(A, B, C)$ where $A=0$, which is disallowed. Hence, we must have, for each $i$, an index $j_{i}$ such that $s_{i, j_{i}} \neq 0$. But this implies, by the above equations, that $m_{i}=f_{j_{i}}$, which itself implies that we must have two values $i_{0}$ and $i_{1}$ such that $m_{i_{0}}=m_{i_{1}}$. This contradicts the fact that all the messages should be distinct.

We conclude that the output of the adversary cannot correspond to a set of polynomial equations which hold for all possible variable assignments. Thus, the adversary must win, or tell it is in a simulation, via a specific assignment to the variables.

PROPERTY Two: If the adversary learned it was interacting in a simulated game, there would exist two different polynomials $P_{1}$ and $P_{2}$ on one 
of the lists such that $P_{1}=P_{2}$ for a specific assignment of values to the variables. We note, that $P_{1}$ cannot equal $P_{2}$ identically by construction of the oracles. Since $\operatorname{deg}\left(P_{1}\right), \operatorname{deg}\left(P_{2}\right) \leq \mathrm{d}_{\max }$ we find that the probability of an assignment being such that $P_{1}=P_{2}$ is bounded by $\mathrm{d}_{\max } / p$ [29][Lemma 1]. Thus, the probability that this happens is bounded by $O\left(n^{2} \cdot \mathrm{d}_{\max } / p\right)$, since the size of the lists are bounded by $n$.

Property Three: In a similar way, we need to bound the probability of the adversary outputing a set of elements, whose associated polynomials satisfy the required equations, for a specific assignment of values to the variables. We have that the total degree of the set of equations is bounded by $9 q_{O}+2=\mathrm{d}_{\max }+1$, and we have $2 \cdot\left(q_{O}+1\right)$ such equations to satisfy. Thus, the probability of Property Three holding is bounded by $O\left(\left(q_{O} / p\right)^{q_{O}}\right)$, i.e. vanishingly small.

In conclusion, the probability that an adversary breaks the E-LRSW assumption in the GGM is bounded by $O\left(n^{2} \cdot q_{O} / p\right)$. 